\title{
Transient Spectroscopic Dynamics of Excitons and Polarons in P3HT:FLR Blend
}

\author{
Jessica Patel ${ }^{1 \mathrm{a}}$, Mihirsinh Chauhan ${ }^{2 \mathrm{a}, \#}$, Abhishek Sharma ${ }^{3,4, b}$, Nikita Vashistha ${ }^{6, b}$, Umesh K \\ Dwivedi $^{5}$, Mahesh Kumar ${ }^{6}$, Brijesh Tripathi ${ }^{*}$, Manoj Kumar Pandey ${ }^{1 *}$, J. P. Tiwari ${ }^{3,4^{*}}$ \\ ${ }^{1}$ Department of Science, School of Technology, Pandit Deendayal Energy University, Gandhinagar - 382007, India. \\ ${ }^{2}$ Department of Solar Energy, School of Technology, Pandit Deendayal Energy University, Gandhinagar - 382007, \\ India \\ ${ }^{3}$ Advanced Materials and Devices Metrology Division (Photovoltaic Metrology Section), CSIR-National Physical \\ Laboratory, NewDelhi,11002, \\ ${ }^{4}$ Academy of Scientific and Innovative Research (AcSIR), CSIR-HRDC Campus, Ghaziabad 201002, India \\ ${ }^{5}$ Department of Applied Physics, Amity School of Applied Sciences, Amity Universiy Rajasthan, Jaipur 303 002, \\ India \\ ${ }^{6}$ Ultrafast Optoelectronics and Terahertz Photonics Lab, Advanced Materials and Devices Metrology Division, \\ CSIR-National Physical Laboratory, New Delhi-110012, India \\ ${ }^{\#}$ Current Address: Soft Matter Optoelectronics, University of Bayreuth, 95440 Bayreuth, Germany
}

*Corresponding authors

Dr.J.P.Tiwari, E-mail: jai_ti2002@yahoo.com, tiwarijp@nplindia.org

Dr. Manoj Kumar Pandey, Email: manoj.pandey@ sot.pdpu.ac.in

Dr. Brijesh Tripathi, Email: brijesh.tripathi@sse.pdpu.ac.in

$\mathrm{a}, \mathrm{b}$ The authors have contributed equally 


\section{SUPPORTING INFORMATION}

\section{S1: Steady-State Absorption and Photoluminescence Spectra:}
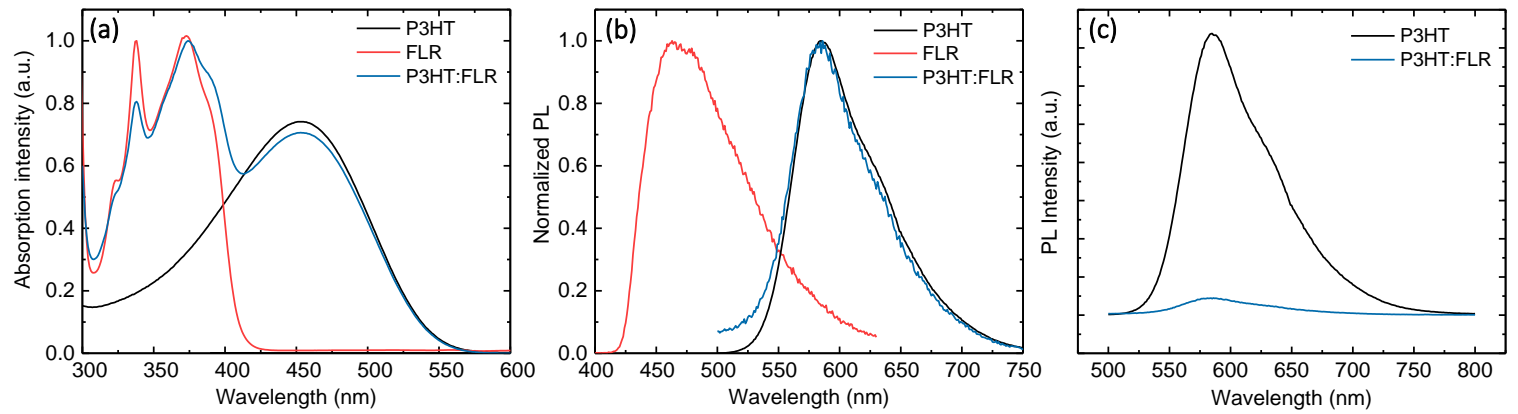

Figure S 1. (a) Absorption spectra, (b) Photoluminescence spectra of P3HT, FLR and P3HT:FLR blend, (c) Photoluminescence spectra of P3HT ad P3HT:FLR blend showing quenching.

Figure S1a shows the steady state absorption spectra of pristine P3HT, FLR and P3HT:FLR blend. The pristine P3HT and FLR shows the absorption peaks at $453 \mathrm{~nm}$ and $294 \mathrm{~nm}, 337 \mathrm{~nm}$, $373 \mathrm{~nm}$ respectively. The P3HT:FLR blend shows the absorption peaks in superposition to individual components. Figure S1b shows the photoluminescence spectra with emission peaks of P3HT and FLR at $600 \mathrm{~nm}$ and $463 \mathrm{~nm}$ respectively. The P3HT:FLR blend shows the emission peak similar to P3HT. Figure S1c shows the PL spectra of pristine P3HT and P3HT:FLR blend. The relative quenching efficiency ${ }^{1}$ is calculated from:

$$
Q=1-\frac{\int P L_{\text {quencher }} d \lambda}{P L_{\text {pristine }} d \lambda}
$$

The relative quenching efficiency (Q) is 94\% for P3HT:FLR blend in chlorobenzene solvent ${ }^{2}$. 


\section{S2: Transient absorption data and spectral analysis:}
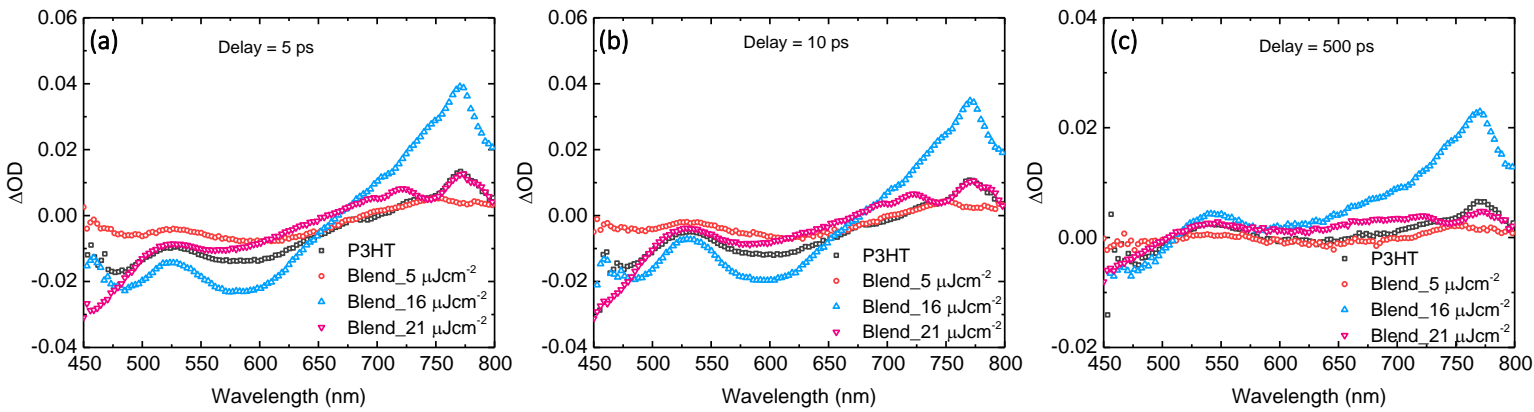

Figure S 2. (a-c) The non-normalized transient absorption response ( $\Delta \mathrm{OD})$ of pristine P3HT and P3HT:FLR blend at various delay times for GSB region.
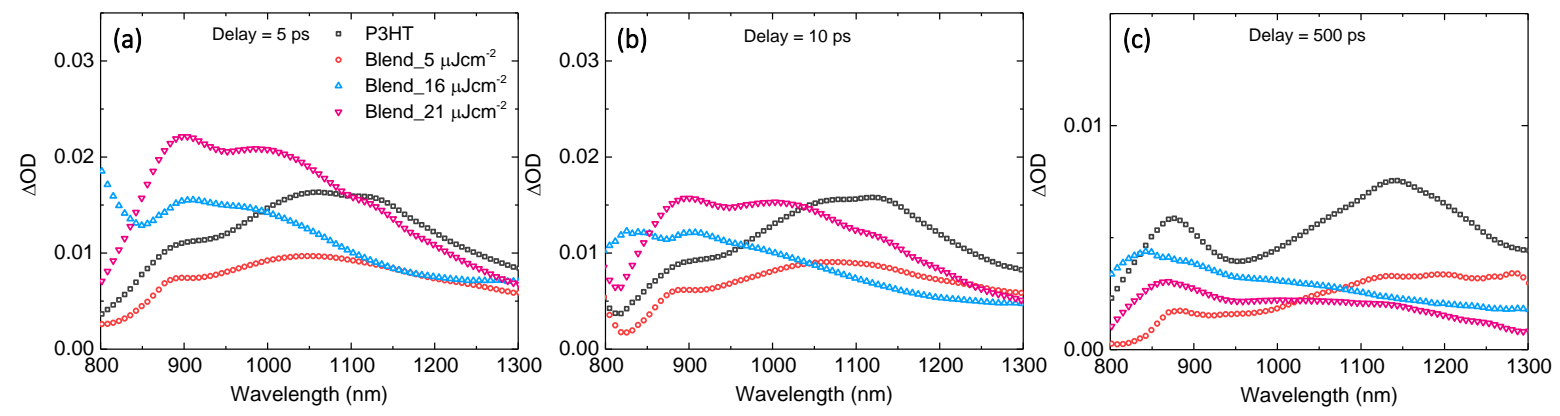

Figure S 3. (a-c) The non-normalized transient absorption response ( $\triangle \mathrm{OD})$ of pristine P3HT and P3HT:FLR at various time delays. The normalized spectra are shown in Figure 2 of main article.

The transient absorption response of pristine P3HT and P3HT:FLR blend in NIR region, photoexcited at $425 \mathrm{~nm}$ with different fluence energies are shown in Figure S3 (a-c). The normalized transient absorption data are shown in Figure 2 in the main article. 

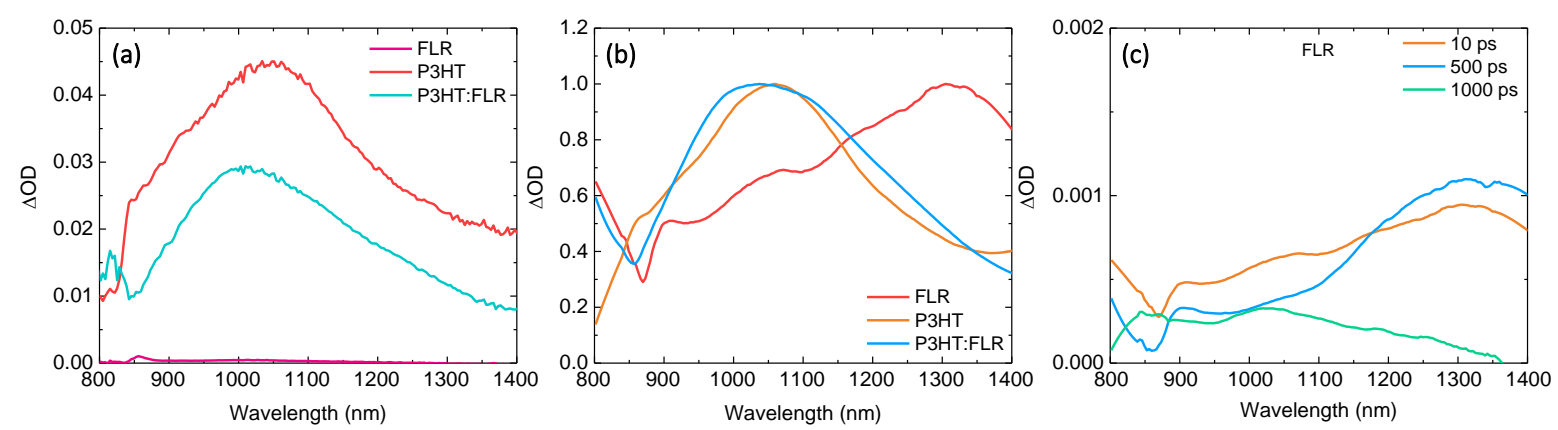

Figure S 4. (a) The non-normalized and (b) normalized transient absorption response ( $\Delta \mathrm{OD})$ for pristine FLR, pristine P3HT and P3HT:FLR blend at $10 \mathrm{ps}$ (FLR: photo-excited at $350 \mathrm{~nm}, 16$ $\mu \mathrm{Jcm}^{-2}$ pulse energy; P3HT and P3HT:FLR blend: photo-excited at $425 \mathrm{~nm}, 16 \mu \mathrm{Jcm}^{-2}$ pulse energy), (c) The change in transient absorption response ( $\triangle \mathrm{OD})$ for FLR measured at various pump-probe time delay. The transient absorption data for pristine FLR shows weaker absorption signals as compared to pristine P3HT under the same fluence energy. The absorption crosssection of the excited states in FLR is weaker compared to P3HT (similar to PCBM or SF-PDI2).

The photo-induced charge transfer $(\mathrm{CT})$ rates for pristine FLR, pristine P3HT and P3HT:FLR blend were measured using the broadband near-infrared transient absorption spectroscopy at 16 $\mu \mathrm{Jcm}^{-2}$ fluence energy. The transient absorption response was recorded in the NIR region from $800 \mathrm{~nm}$ to $1400 \mathrm{~nm}$. With the same fluence energy, there is no transient absorption signal above the noise limit for pristine FLR. The transient absorption response for FLR can be observed when it is excited at $370 \mathrm{~nm}$. Also, the intensity of the transient absorption signal is very weak. Figure S4a shows the transient absorption response of pristine FLR is only $\sim 1 \times 10^{-3}$ as compared to pristine P3HT $\left(\sim 4 \times 10^{-2}\right)$ and P3HT:FLR blend $\left(\sim 3 \times 10^{-2}\right)$ when excited at the same fluence energy. This shows that the absorption cross-section of the excited states (for excitons and polarons) in FLR is an order of magnitude lower than P3HT and therefore their presence in the blend does not significantly affect the analysis. This can be further confirmed by the overlapping transient absorption response of P3HT:FLR at early time delay. The spectra should look different if the excitons and polaron in FLR have comparable absorption cross-section to that in P3HT 
(see Figure S4b). This type of similar behavior of acceptor can be observed for SF-PDI ${ }_{2}^{3}$ and $\mathrm{PCBM}^{4}$. Therefore, the excited states in the FLR domain can be neglected in the analysis described in the main text. The pristine P3HT shows the broad excited state absorption (ESA) at $\sim 1050 \mathrm{~nm}$ which can be attributed to the singlet excitons. The transient absorption response of P3HT:FLR blend shows the similar ESA band with a blue shift. The new feature corresponding to the blue shift refers to the hole polaron feature. For the pristine FLR, there is a broad band near $1020 \mathrm{~nm}$ which overlaps with the P3HT:FLR blend absorption feature. The change in the transient absorption response for FLR at various time delays are shown in Figure S4c. At shorter time delays, there is a broad absorption band near $\sim 1300 \mathrm{~nm}$. This can be attributed to the formation of FLR cation. This may be formed from the dissociation of the excited states in the FLR domain as long-lived charge carriers. In previous pristine PCBM studies, it has been revealed that there is enhanced absorption in the visible region due to PCBM domains attributed by the intermolecular CT transitions which is correlated with the photocurrent generation. ${ }^{5}$ However, there is decrease in intensity of band $\sim 1300 \mathrm{~nm}$ with longer time delay which may be due to the bimolecular recombination of the FLR cation. 

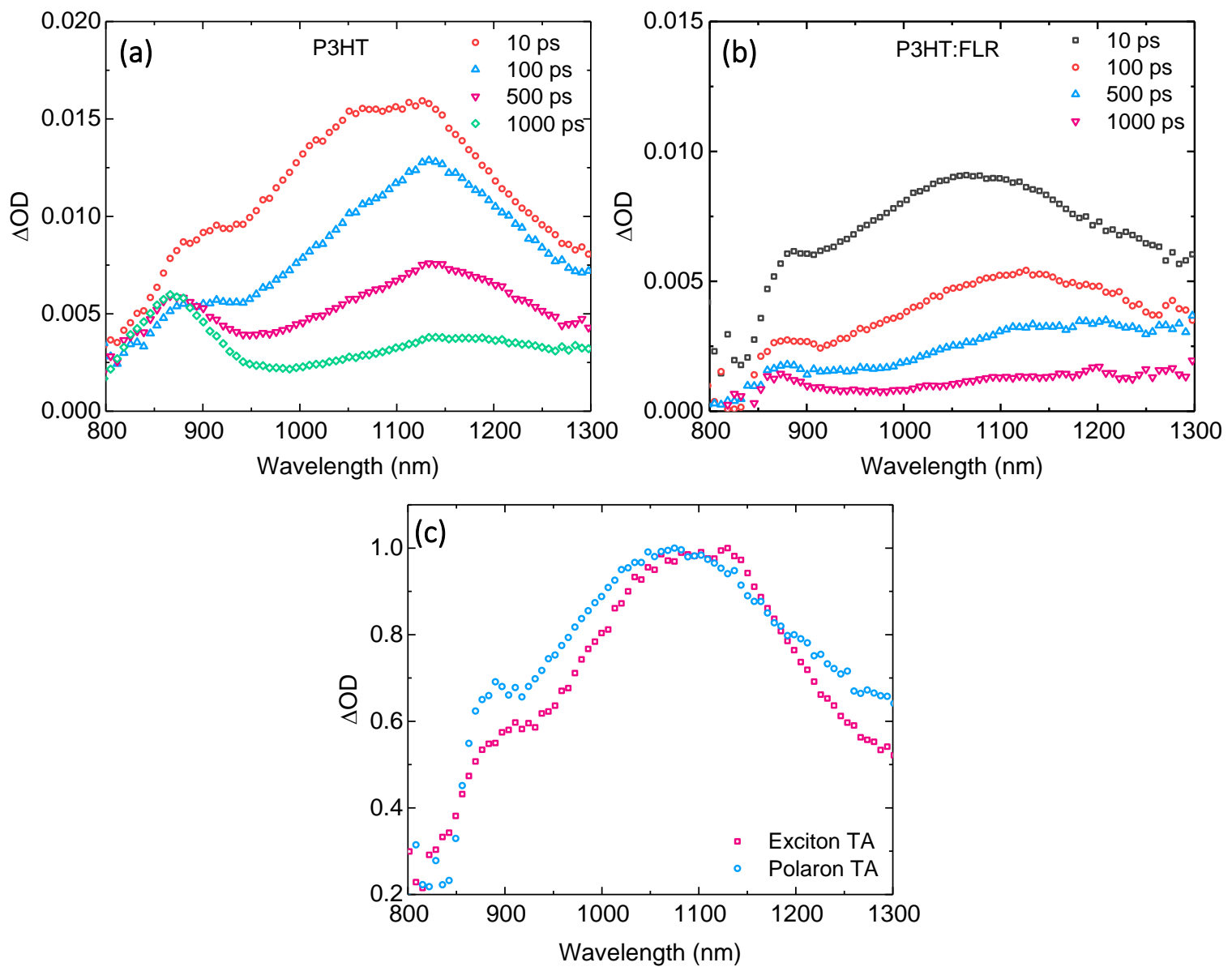

Figure S 5. (a, b) Non-normalized transient absorption response of pristine P3HT and P3HT:FLR excited at $425 \mathrm{~nm}$ with $5 \mu \mathrm{Jcm}^{-2}$ per pulse, (c) The estimated transient absorption response of P3HT singlet excitons and polaron (normalized to the peak signal at $\sim 1100 \mathrm{~nm}$ )

Figure S5 shows the measured transient absorption data of pristine P3HT and P3HT:FLR blend measured at $5 \mu \mathrm{Jcm}^{-2}$. Here, we differentiate the transient absorption response of singlet excitons and polaron in P3HT. The excitons separate into free charges at the donor-acceptor interface, therefore the transient absorption response for pristine P3HT (Figure S5a) are mostly due to singlet excitons. There is slight change in the spectral evolution of pristine P3HT in the range of $830 \mathrm{~nm}$ to $950 \mathrm{~nm}$. This changes may be either due to (a) lower stimulated emission due to movement of excitons to the less emissive sites, (b) some excitons that separate at disordered regions forming polaron. Therefore, the excitons are the dominant species in pristine P3HT and 
the transient absorption response are attributed to the excitons (Figure S5c). At early times, the singlet excitons are dominant species in P3HT:FLR blend and at longer time delay there is balance between excitons, free charge carriers and charge transfer excitons. It is to be noted that free charge carriers and charge transfer excitons have polaron transient absorption signal. Therefore, it is difficult to differentiate between polaron response in the P3HT:FLR blend.

\section{S3: Transient spectra normalization to demonstrate $\triangle \triangle O D:$}

The transient absorption measurements for pristine P3HT and P3HT:FLR blend were taken in chlorobenzene solvents with identical experimental conditions. To determine the polaron feature in the spectra accurately, the $\Delta \mathrm{OD}$ were analyzed at different time delays. In the transient absorption response of P3HT:FLR blend, there is contribution from free and bound polymer. The bound polymer will give the characteristic polaron feature. The transient absorption spectra are normalized to eliminate the free exciton contribution from the spectra at different time delays by observing the exciton feature on the longer wavelength edge. The difference between these spectra have been utilized to develop $\Delta \Delta \mathrm{OD}$ spectra which determines the polaron feature only which arises from the bound polymer. This method has been followed to generate spectra as shown in Figure S6. 

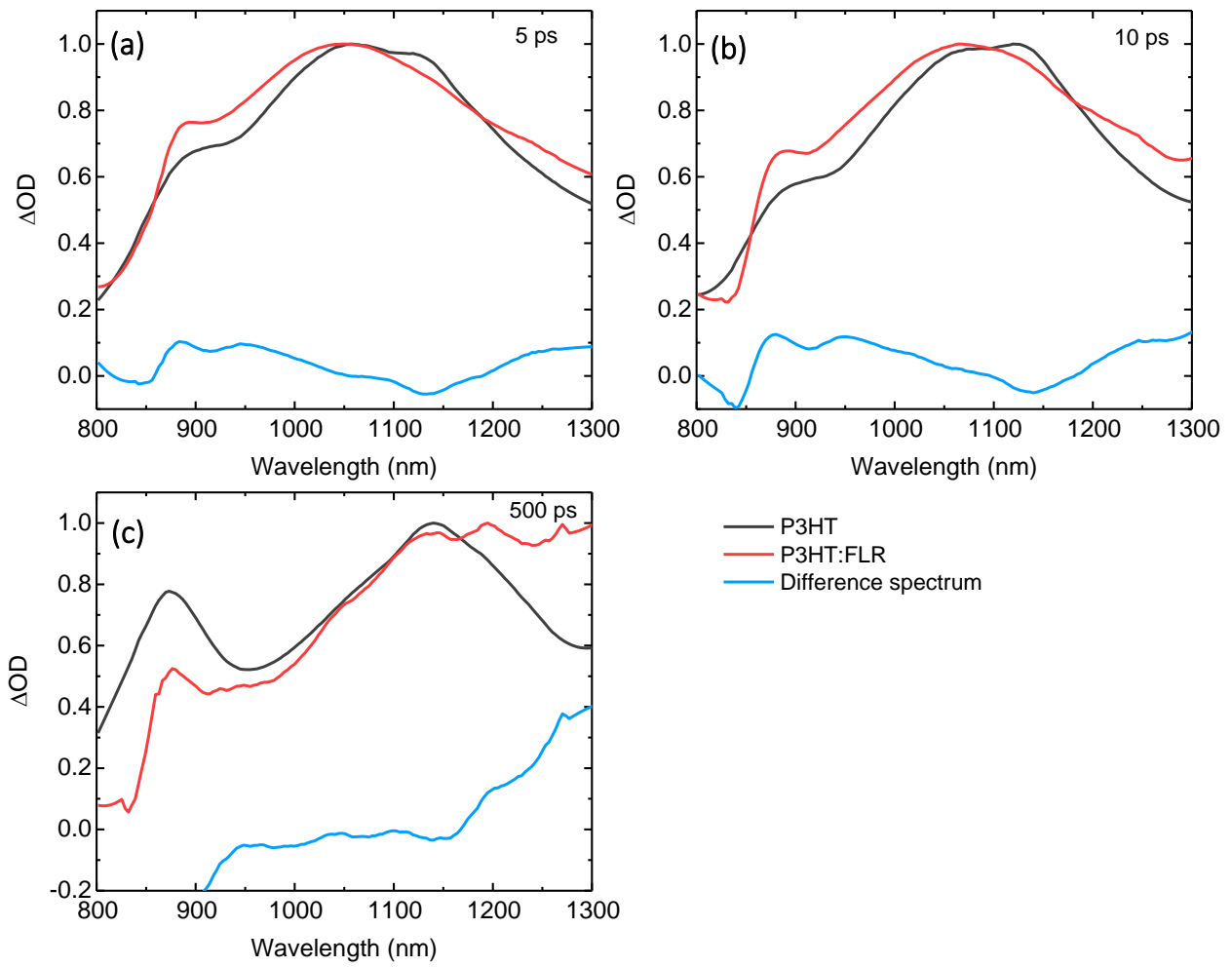

- P3HT

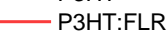

Difference spectrum

Figure S 6. $\triangle \Delta \mathrm{OD}$ spectra of pristine $\mathrm{P} 3 \mathrm{HT}$, P3HT:FLR blend and difference spectrum at different delay time

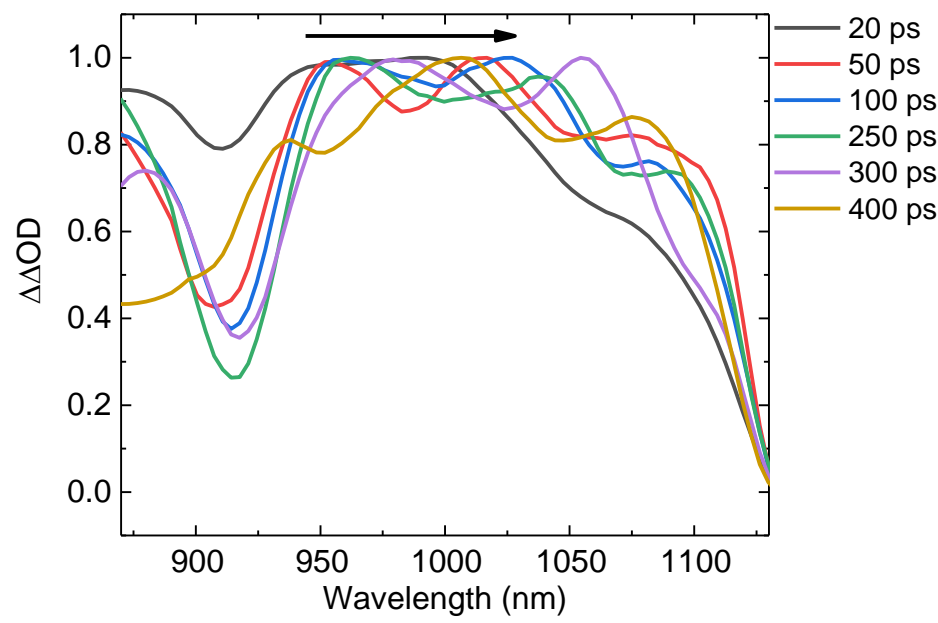

Figure S 7. $\triangle \Delta \mathrm{OD}$ (normalized) spectrum for P3HT:FLR blend with time series showing spectral evolution of the polaron feature 


\section{S4: Excitation Intensity Dependence of Singlet Exciton Decay:}

Figure S8 shows the excitation intensity dependence of transient absorption signals at $1000 \mathrm{~nm}$ of P3HT:FLR blend. The decay fraction at $1000 \mathrm{~nm}$ is attributed to singlet excitons. The transient absorption signals at $470 \mathrm{~nm}$ is attributed to the photo-bleaching of ground state of P3HT (Figure S9 bottom panel of stack). The dynamics at $470 \mathrm{~nm}$ and $1000 \mathrm{~nm}$ were independent of the excitation intensities from 5,16 and $21 \mu \mathrm{Jcm}^{-2}$ suggesting that the quenching of singlet excitons due to charge transfer is faster than other processes such as singlet excitonexciton annihilation. Also, there is no change in the ground state photo-bleaching signals at 470 $\mathrm{nm}$ which indicates that singlet excitons do not return to the ground state. Thus, almost all the singlet excitons can be separated into the polymer polarons. These results can be consistent with the quenching efficiency of this blend.

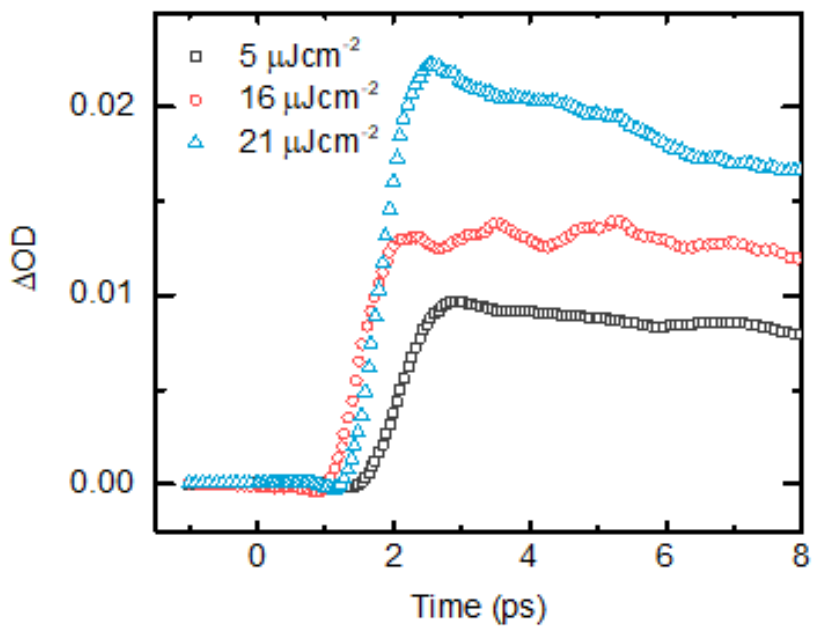

Figure S 8. Transient absorption signals at $1000 \mathrm{~nm}$ at different fluence energy to understand excitation intensity dependence of singlet exciton decay. 


\section{S5: Excitation Intensity Dependence for Charge Recombination:}

The decay dynamics of three transient signals in P3HT:FLR blend on a time scale of nanoseconds: the ground-state photo-bleaching $(470 \mathrm{~nm})$, P3HT polarons $(870 \mathrm{~nm}, 1140 \mathrm{~nm})$ under different excitation intensities are shown in Figure S9. The decay dynamics at these three characteristics bands was independent of the excitation intensities ranging from $5,16,21 \mu \mathrm{Jcm}^{-2}$, suggesting geminate recombination of bound radical pairs.
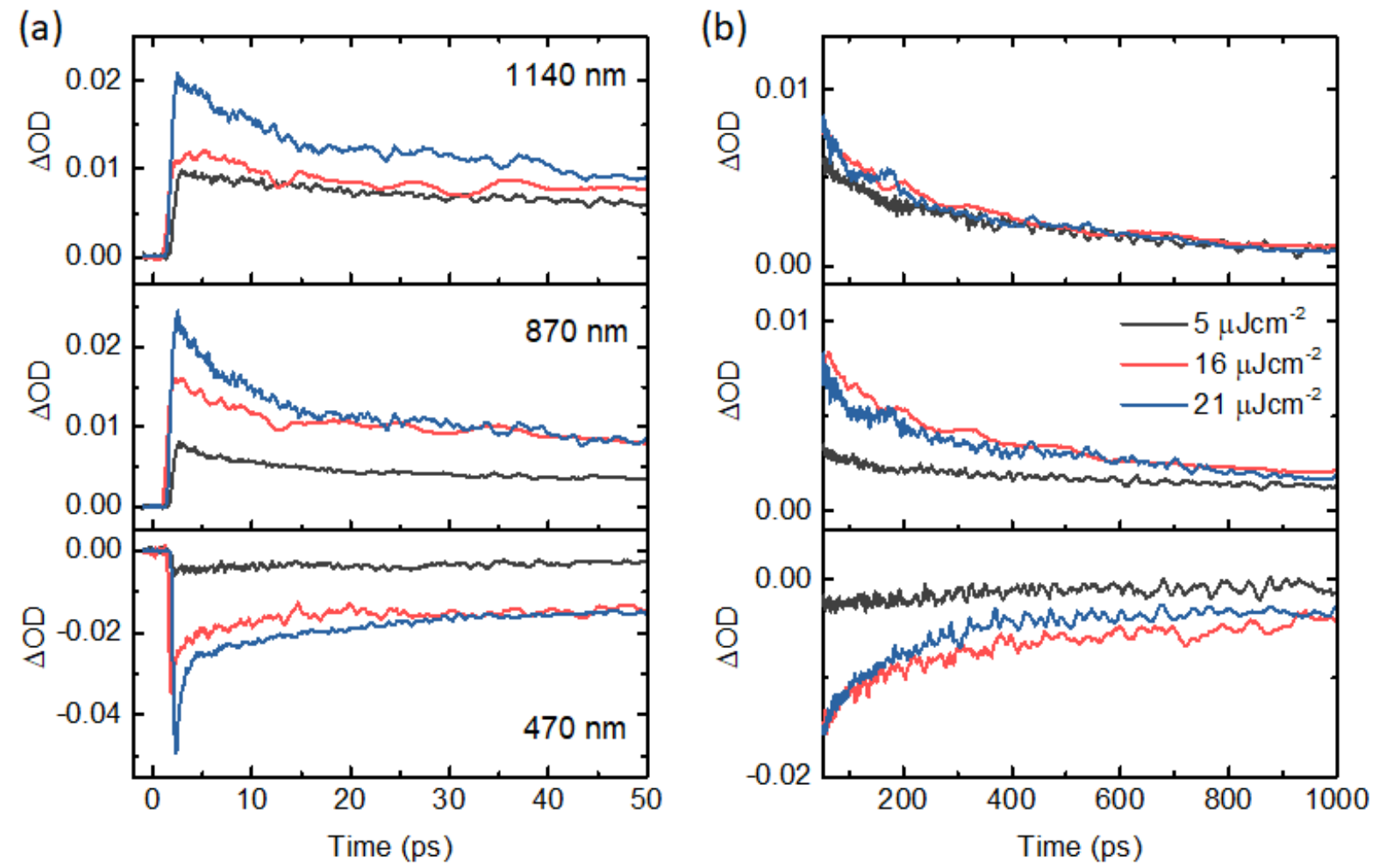

Figure S 9. Transient absorption decays of P3HT:FLR blend excited at $425 \mathrm{~nm}$ measured at (a) $1140 \mathrm{~nm}$ (upper stack), $870 \mathrm{~nm}$ (bottom stack) and $470 \mathrm{~nm}$ (lower stack) for different fluence energies for first $50 \mathrm{ps}$ and (b) for longer time scale, respectively. 


\section{S6: Extended description of the higher polaron signal for the blend with a longer lifetime:}

There is rise in polaron signal with the increase in delay times (Figure 4). To give more clarity on this, we regenerate TA spectra published previously by our group for P3HT:FLR blend in chlorobenzene (CB), 1,2-dichlorobenzene (ODCB) and chloroform (CF) solvents at $16 \mu \mathrm{Jcm}^{-2}$ as shown in Figure S10. It shows that there is change in the polaron signal for different solvents. The polaron signal for blend with a longer lifetime is also observed for blend in CF. But the polaron signal is different for the blend in ODCB. This may be due to different morphology by the solvents. Similar study is done by Hinrichsen et al., for P3TEA:SF-PDI 2 blend system. They also observed longer lifetime of polaron for P3TEA: SF-PDI 2 blend as compared to P3TEA:PCBM blend system. ${ }^{3}$ This longer lifetime of polarons for non-fullerene systems may be due to: (1) the low degree of electronic disorder at donor-acceptor interface or (2) slow transient rate of charge transfer excitons to triplet exciton. Therefore, our results presented here indicate that longer lifetime of polaron signal is real and may increase the charge separation up to hundreds of picoseconds (Information has been added in SI: Page no. S11). 


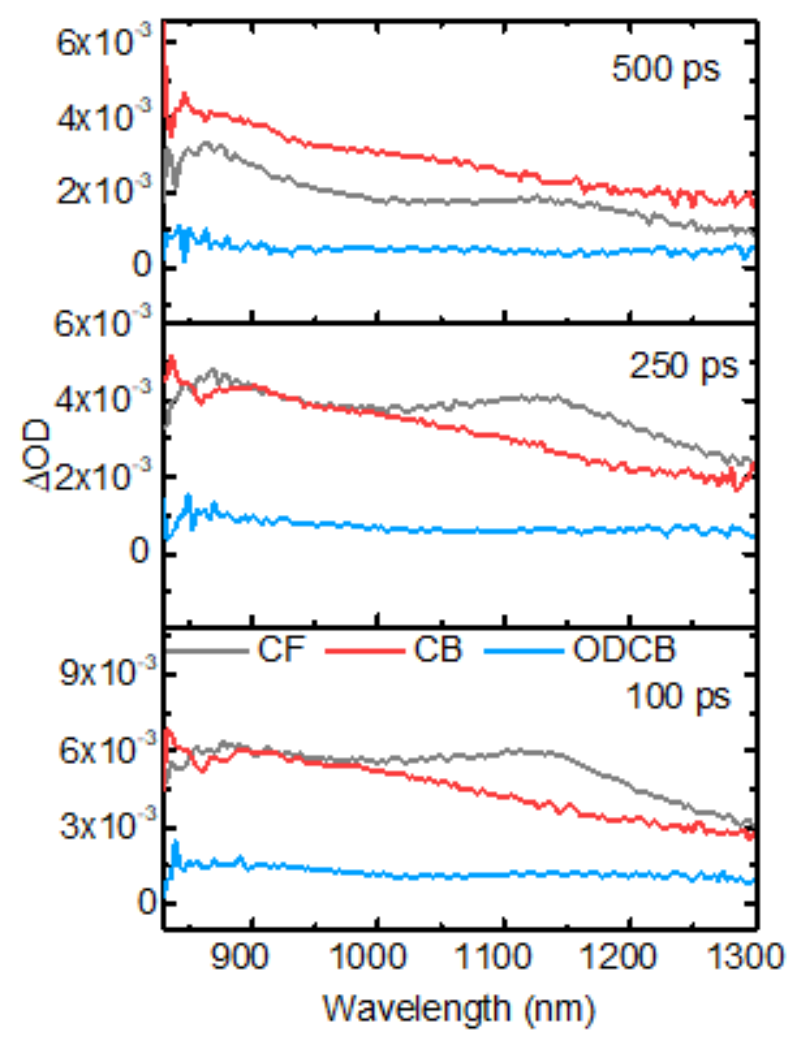

Figure S 10: Transient absorption spectra for P3HT:FLR blend at room temperature (photoexcited at $425 \mathrm{~nm}$ ) for different solution

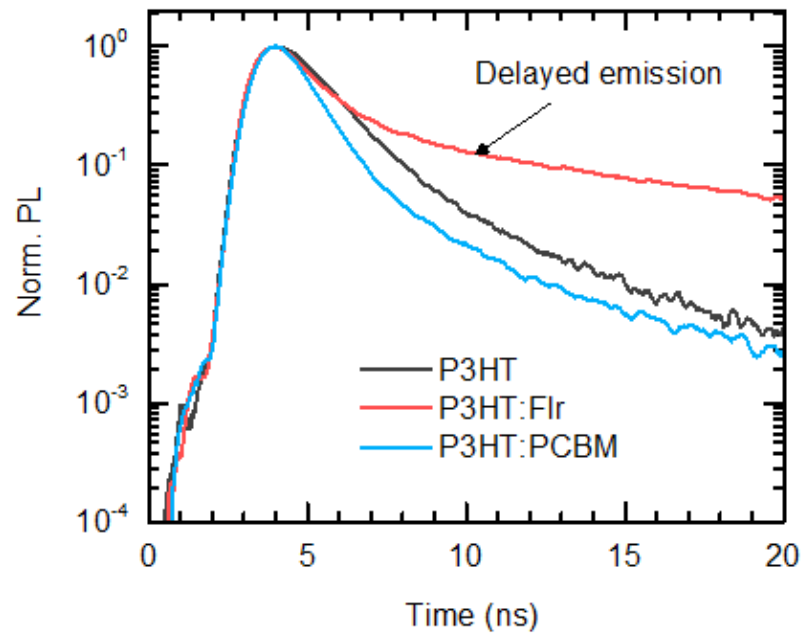

Figure S 11. Time-resolved PL decay of P3HT, P3HT:FLR and P3HT:PCBM blend under pulsed $480 \mathrm{~nm}$ excitations. 


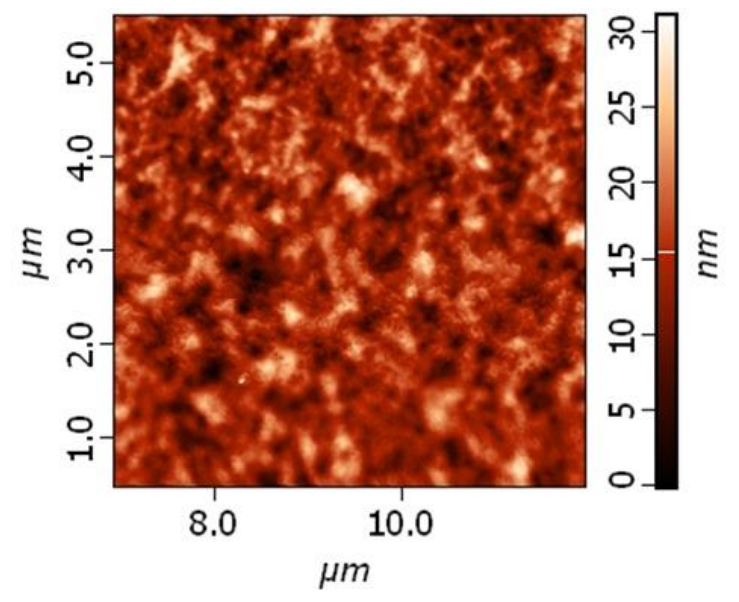

Figure S12: AFM images of P3HT:FLR film (Average roughness is $~ 3.5 \mathrm{~nm}$ ).

(a)

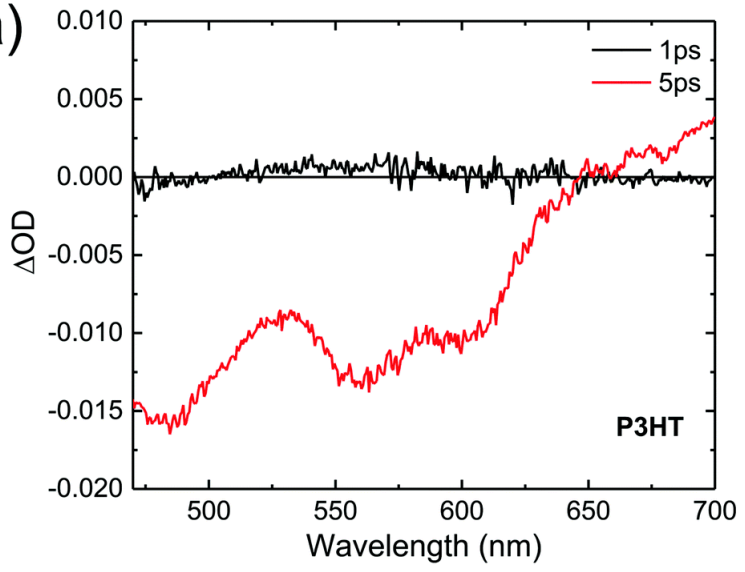

(C)

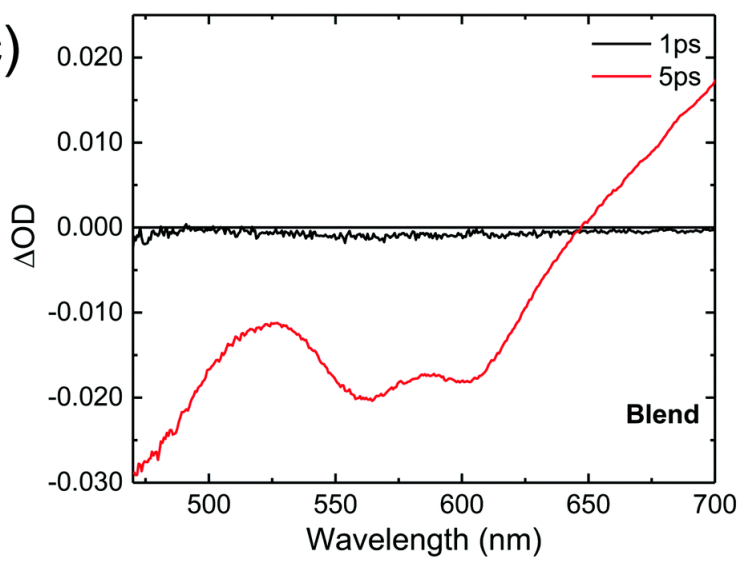

(b)

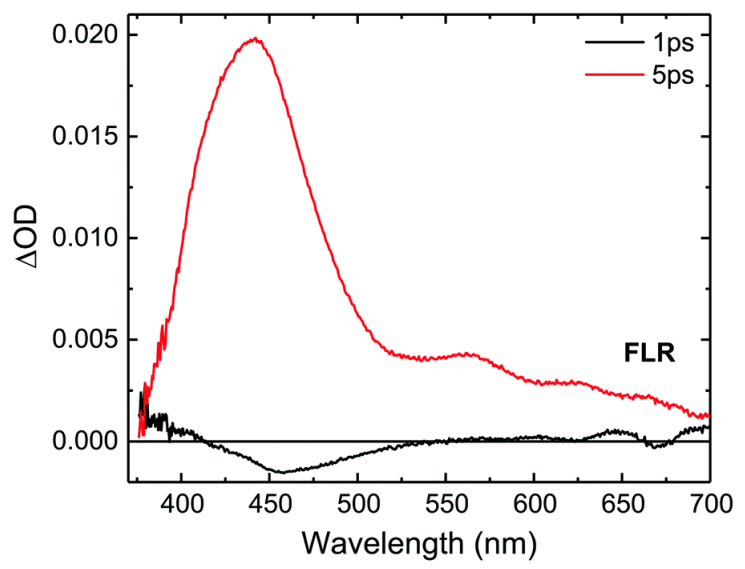

(d)

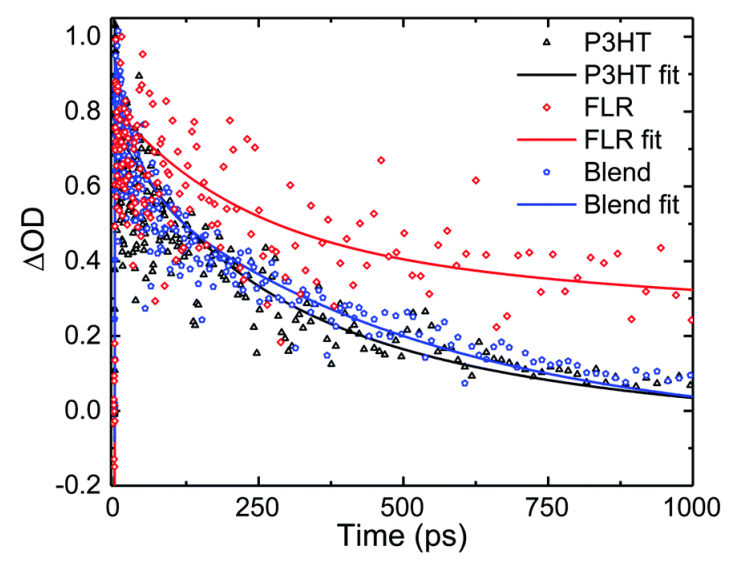

Figure S 13: A spectra at 1 ps and 5 ps of (a) pristine P3HT, (b) FLR, and (c) the P3HT:FLR blend in THF and (d) kinetic curves of pristine P3HT, pristine FLR and the P3HT:FLR blend probed at $1040 \mathrm{~nm}$ to study the singlet exciton formation ${ }^{2}$ (Reproduced by permission of the PCCP Owner Societies). The TA spectra of P3HT:FLR and the kinetics studies for pristine P3HT and FLR and their blend elucidate their interfacial charge dynamics. 
Table S1: Kinetic time constants of P3HT:FLR blends at different fluence energy.

\begin{tabular}{|c|c|c|c|}
\hline Parameters & $\mathbf{5} \boldsymbol{\mu J} \mathbf{J c m}-\mathbf{2}$ & $\mathbf{1 6} \boldsymbol{\mu J c m - 2}$ & $\mathbf{2 1} \boldsymbol{\mu} \mathbf{J c m}-\mathbf{2}$ \\
\hline A1 & 0.14 & 0.29 & 0.22 \\
\hline$\tau 1(\mathrm{ps})$ & 12 & 8 & 5 \\
\hline A2 & 0.37 & 0.4 & 0.5 \\
\hline$\tau 2(\mathrm{ps})$ & 58 & 158 & 40 \\
\hline A3 & 0.49 & 0.31 & 0.28 \\
\hline$\tau 3(\mathrm{ps})$ & 555 & 700 & 456 \\
\hline
\end{tabular}

\section{References:}

1. Lin, J. D.; Mikhnenko, O. V.; Chen, J.; Masri, Z.; Ruseckas, A.; Mikhailovsky, A.; Raab, R. P.; Liu, J.; Blom, P. W.; Loi, M. A., Systematic study of exciton diffusion length in organic semiconductors by six experimental methods. Mater. Horiz. 2014, 1 (2), 280-285.

2. $\quad$ Patel, J.; Sharma, A.; Chauhan, M.; Aatif, M.; Vashistha, N.; Kumar, M.; Tripathi, B.; Chand, S.; Tiwari, J.; Pandey, M. K., Understanding charge carrier dynamics in a P3HT: FLR blend. Phys. Chem. Chem. Phys. 2019, 21 (5), 2771-2782.

3. Hinrichsen, T. F.; Chan, C. C.; Ma, C.; Paleček, D.; Gillett, A.; Chen, S.; Zou, X.; Zhang, G.; Yip, H.-L.; Wong, K. S., Long-lived and disorder-free charge transfer states enable endothermic charge separation in efficient non-fullerene organic solar cells. Nat. Commun. 2020, 11 (1), 1-10.

4. Roy, P.; Jha, A.; Dasgupta, J., Photoinduced charge generation rates in soluble P3HT: PCBM nano-aggregates predict the solvent-dependent film morphology. Nanoscale 2016, 8 (5), 2768-2777.

5. Yamamoto, S.; Guo, J.; Ohkita, H.; Ito, S., Formation of Methanofullerene Cation in Bulk Heterojunction Polymer Solar Cells Studied by Transient Absorption Spectroscopy. Adv. Funct. Mater. 2008, 18 (17), 2555-2562. 